\title{
On a Class of Supereulerian Digraphs
}

\author{
Khalid A. Alsatami' ${ }^{1}$ Xindong Zhang 2 , Juan Liu' ${ }^{2}$, Hong-Jian Lai ${ }^{3}$ \\ ${ }^{1}$ Department of Mathematics, College of Science, Qassim University, Buraydah, KSA \\ ${ }^{2}$ College of Mathematics Sciences, Xinjiang Normal University, Urumqi, China \\ ${ }^{3}$ Department of Mathematics, West Virginia University, Morgantown, WV, USA \\ Email:kaf043@gmail.com, liaoyuan1126@163.com, liujuan1999@126.com, hongjianlai@gmail.com
}

Received 4 January 2016; accepted 26 February 2016; published 29 February 2016

Copyright (C) 2016 by authors and Scientific Research Publishing Inc.

This work is licensed under the Creative Commons Attribution International License (CC BY).

http://creativecommons.org/licenses/by/4.0/

(c) (i) Open Access

\section{Abstract}

The 2-sum of two digraphs $D_{1}$ and $D_{2}$, denoted $D_{1} \oplus_{2} D_{2}$, is the digraph obtained from the disjoint union of $D_{1}$ and $D_{2}$ by identifying an arc in $D_{1}$ with an arc in $D_{2}$. A digraph $D$ is supereulerian if $D$ contains a spanning eulerian subdigraph. It has been noted that the 2-sum of two supereulerian (or even hamiltonian) digraphs may not be supereulerian. We obtain several sufficient conditions on $D_{1}$ and $D_{2}$ for $D_{1} \oplus_{2} D_{2}$ to be supereulerian. In particular, we show that if $D_{1}$ and $D_{2}$ are symmetrically connected or partially symmetric, then $D_{1} \oplus_{2} D_{2}$ is supereulerian.

\section{Keywords}

Supereulerian, Digraph 2-Sums, Arc-Strong-Connectivity, Hamiltonian-Connected Digraphs

\section{Introduction}

We consider finite graphs and digraphs, and undefined terms and notations will follow [1] for graphs and [2] for digraphs. Throughout this paper, the notation $(u, v)$ denotes an arc oriented from $u$ to $v$. A digraph $D$ is strict if it contains no parallel arcs nor loops; and is symmetric if for any vertices $u, v \in V(D)$, if $(u, v) \in A(D)$, then $(v, u) \in A(D)$. If two arcs of $D$ have a common vertex, we say that these two arcs are adjacent in $D$. A directed path in a digraph $D$ from a vertex $u$ to a vertex $v$ is called a $(u, v)$-dipath. To emphasize the distinction between graphs and digraphs, a directed cycle or path in a digraph is often referred as a dicycle or dipath. A dipath $P$ is a hamiltonian dipath if $V(P)=V(D)$. A digraph $D$ is hamiltonian if $D$ contains a hamiltonian dicycle. An $(x, y)$-hamiltonian dipath is a hamiltonian dipath from $x$ to $y$. A digraph $D$ is hamiltonian-connected if $D$ has an $(x, y)$-hamiltonian dipath for every choice of distinct vertices $x, y \in V(D)$.

As in [2], $\lambda(D)$ denotes the arc-strong-connectivity of $D$. A digraph $D$ is strong if and only if $\lambda(D) \geq 1$. For $X, Y \subseteq V(D)$, we define 


$$
(X, Y)_{D}=\{(x, y) \in A(D): x \in X \text { and } y \in Y\} \text {; and } \partial_{D}^{+}(X)=(X, V(D)-X)_{D} .
$$

For a subset $A^{\prime} \subseteq A(D)$, the subdigraph arc-induced by $A^{\prime}$ is the digraph $D\left[A^{\prime}\right]=\left(V^{\prime}, A^{\prime}\right)$, where $V^{\prime}$ is the set of vertices in $V$ which are incident with at least one arc in $A^{\prime}$.

Let

$$
d_{D}^{+}(X)=\left|\partial_{D}^{+}(X)\right| \text {, and } d_{D}^{-}(X)=\left|\partial_{D}^{-}(X)\right| \cdot
$$

When $X=\{v\}$, we write $d_{D}^{+}(v)=\left|\partial_{D}^{+}\{v\}\right|$ and $d_{D}^{-}(v)=\left|\partial_{D}^{-}\{v\}\right|$. Let $N_{D}^{+}(v)=\{u \in V(D)-v:(v, u) \in A(D)\}$ and $N_{D}^{-}(v)=\{u \in V(D)-v:(u, v) \in A(D)\}$ denote the out-neighbourhood and in-neighbourhood of $v$ in $D$, respectively. Vertices in $N_{D}^{+}(v), N_{D}^{-}(v)$ are called the out-neighbours, in-neighbours of $v$. Thus for a digraph $D$ and an integer $k \geq 0$,

$$
\lambda(D) \geq k \text { if and only if for any } W \text { with } \varnothing \neq W \subset V(D),\left|\partial_{D}^{+}(W)\right| \geq k .
$$

Boesch, Suffel, and Tindell [3] in 1977 proposed the supereulerian problem, which seeks to characterize graphs that have spanning eulerian subgraphs. They indicated that this problem would be very difficult. Pulleyblank [4] later in 1979 proved that determining whether a graph is supereulerian, even within planar graphs, is NPcomplete. Catlin [5] in 1992 presented the first survey on supereulerian graphs. Chen et al. [6] surveyed the reduction method associated with the supereulerian problem and their applications. An updated survey presenting the more recent developments can be found in [7].

It is natural to consider the supereulerian problem in digraphs. A digraph $D$ is eulerian if it contains a closed ditrail $W$ such that $A(W)=A(D)$, or, equivalently, if $D$ is strong and for any $v \in V(D), d_{D}^{+}(v)=d_{D}^{-}(v)$. A digraph $D$ is supereulerian if $D$ contains a closed ditrail $W$ such that $V(W)=V(D)$, or, equivalently, if $D$ contains a spanning eulerian subdigraph. Some recent developments on supereulerian digraphs are given in [8]-[12].

A central problem is to determine or characterize supereulerian digraphs. In Section 2, the 2-sum $D_{1} \oplus_{2} D_{2}$ of two digraphs $D_{1}$ and $D_{2}$ is defined, and some basic properties of 2-sums are discussed. We will observe that a 2-sum of two supereulerian (or even hamiltonian) digraphs may not be supereulerian. Thus it is natural to seek sufficient conditions on $D_{1}$ and $D_{2}$ for the 2-sum of $D_{1}$ and $D_{2}$ to be supereulerian. In the last section, we will present several sufficient conditions for supereulerian 2-sums of digraphs. In particular, we show that if $D_{1}$ and $D_{2}$ are either symmetrically connected or partially symmetric (to be defined in Section 3), then $D_{1} \oplus_{2} D_{2}$ is supereulerian.

\section{The 2-Sums of Digraphs}

The definition and some elementary properties of the 2-sums of digraphs are presented in this section. A digraph is nontrivial if it contains at least one arc. Throughout this section, all digraphs are assumed to be nontrivial.

Definition 2.1 Let $D_{1}$ and $D_{2}$ be two vertex disjoint digraphs, and let $a_{1}=\left(v_{11}, v_{12}\right) \in A\left(D_{1}\right)$ and $a_{2}=\left(v_{21}, v_{22}\right) \in A\left(D_{2}\right)$ be two distinguished arcs. The 2-sum $D_{1} \oplus_{a_{1}, a_{2}} D_{2}$ of $D_{1}$ and $D_{2}$ with base arcs $a_{1}$ and $a_{2}$ is obtained from the union of $D_{1}$ and $D_{2}-a_{2}$ by identifying $v_{11}$ with $v_{21}$ and $v_{12}$ with $v_{22}$, respectively. When the arcs $a_{1}$ and $a_{2}$ are not emphasized or is understood from the context, we often use $D_{1} \oplus_{2} D_{2}$ for $D_{1} \oplus_{a_{1}, a_{2}} D_{2}$.

Lemma 1 Let $D_{1}$ and $D_{2}$ be two vertex disjoint strong digraphs. Then

$$
\lambda\left(D_{1} \oplus_{2} D_{2}\right) \geq \min \left\{\lambda\left(D_{1}\right), \lambda\left(D_{2}\right)\right\} .
$$

Proof. Let $k \geq 0$ be an integer such that $\min \left\{\lambda\left(D_{1}\right), \lambda\left(D_{2}\right)\right\}=k$, and let $\lambda\left(D_{1} \oplus_{2} D_{2}\right)=k^{\prime}$. We shall show that $k^{\prime} \geq k$. By (1), there exists a proper nonempty vertex subset $X \subset V\left(D_{1} \oplus_{2} D_{2}\right)$ such that $\left|\partial_{D_{1} \oplus_{2} D_{2}}^{+}(X)\right|=k^{\prime}$. Let $S=\partial_{D_{1} \oplus_{2} D_{2}}^{+}(X)$. We argue by contradiction and assume that $k^{\prime}<k$.

By Definition 2.1, we have $v_{11}=v_{21} \in V\left(D_{2}\right)$ and $v_{12}=v_{22} \in V\left(D_{2}\right)$ in $D_{1} \oplus_{2} D_{2}$. If $X \cap V\left(D_{1}\right) \neq \varnothing$ and $X \cap V\left(D_{2}\right)=\varnothing$, we obtain that $v_{11}=v_{21} \notin X$ and $v_{12}=v_{22} \notin X$, then $X \subset V\left(D_{1}\right)$ and $S=\partial_{D_{1}}^{+}(X)$. It follows by (1) that $k^{\prime}=|S| \geq \lambda\left(D_{1}\right) \geq k$, contrary to the assumption that $k^{\prime}<k$. Similarly, if $X \cap V\left(D_{1}\right)=\varnothing$ and $X \cap V\left(D_{2}\right) \neq \varnothing$, then $X \subset V\left(D_{2}\right)$ and $S=\partial_{D_{2}}^{+}(X)$, hence a contradiction to the assumption that $k^{\prime}<k$ is obtained from $k^{\prime}=|S| \geq \lambda\left(D_{2}\right) \geq k$. 
Thus, we may assume that $X \cap V\left(D_{1}\right) \neq \varnothing$ and $X \cap V\left(D_{2}\right) \neq \varnothing$. Let $X^{\prime}=X \cap V\left(D_{1}\right)$. Then $X^{\prime}$ is a proper nonempty subset of $V\left(D_{1}\right)$, and $\partial_{D_{1}}^{+}\left(X^{\prime}\right) \subseteq S$. It follows by (1) that $k^{\prime}=|S| \geq\left|\partial_{D_{1}}^{+}\left(X^{\prime}\right)\right| \geq \lambda\left(D_{1}\right) \geq k$ contrary to the assumption that $k^{\prime}<k$.

Example 2.1 The converse of Lemma 1 may not always stand, as indicated by the example below, depicted in Figure 1. Let $V\left(D_{1}\right)=\left\{v_{11}, v_{12}, v_{13}, v_{14}\right\}$ and $V\left(D_{2}\right)=\left\{v_{21}, v_{22}, v_{23}, v_{24}\right\}$. Let

$A\left(D_{1}\right)=\left\{\left(v_{11}, v_{12}\right),\left(v_{13}, v_{12}\right),\left(v_{14}, v_{13}\right),\left(v_{11}, v_{14}\right),\left(v_{11}, v_{13}\right),\left(v_{14}, v_{12}\right)\right\}$ and

$A\left(D_{2}\right)=\left\{\left(v_{21}, v_{22}\right),\left(v_{22}, v_{23}\right),\left(v_{23}, v_{24}\right),\left(v_{24}, v_{21}\right),\left(v_{23}, v_{21}\right),\left(v_{24}, v_{22}\right)\right\}$. Let $a_{1}=\left(v_{11}, v_{12}\right)$ and $a_{2}=\left(v_{21}, v_{22}\right)$.

Then, it is routine to verify that $\lambda\left(D_{1} \oplus_{a_{1}, a_{2}} D_{2}\right) \geq 1$. While $D_{2}$ is strong, the digraph $D_{1}$ contains a vertex $v_{11}$ with $d_{D_{1}}^{-}\left(v_{11}\right)=0$, and so $\lambda\left(D_{1}\right)=0$.

Lemma 2 A digraph $D$ is not supereulerian if for some integer $m>0, V(D)$ has vertex disjoint subsets $\left\{B, B_{1}, \cdots, B_{m}\right\}$ satisfying both of the following:

i) $N_{D}^{-}\left(B_{i}\right) \subseteq B, \forall i \in\{1,2, \cdots, m\}$.

ii) $\left|\partial_{D}^{-}(B)\right| \leq m-1$.

Proof. By contradiction, we assume that both i) and ii) hold and $D$ is supereulerian. Let $S$ be a spanning eulerian subdigraph of $D$, then $B \subset V(S)=V(D)$ and $A(S) \subset A(D)$. Since $S$ is eulerian, for any subset $X \subset V(S)$, it follows that $\left|\partial_{S}^{+}(X)\right|=\left|\partial_{S}^{-}(X)\right|$. Thus, by ii), we conclude that

$$
\left|\partial_{D}^{+}(B) \cap A(S)\right|=\left|\partial_{D}^{-}(B) \cap A(S)\right| \leq\left|\partial_{D}^{-}(B)\right| \leq m-1 .
$$

By i) and by (2), there must be a $B_{j}$ with $j \in\{1,2, \cdots, m\}$ such that $\partial_{D}^{-}\left(B_{j}\right) \cap A(S)=\varnothing$, contrary to the assumption that $V(S)=V(D)$.

Lemma 2 can be applied to find examples of hamiltonian digraphs whose 2-sum is not supereulerian, as shown in Example 2.2 below.

Example 2.2 Let $n_{1}, n_{2} \geq 3$ be integers and $C_{n_{1}}$ and $C_{n_{2}}$ be two vertex disjoint dicycles with length $n_{1}$ and $n_{2}$, respectively. We claim that $C_{n_{1}} \oplus_{2} C_{n_{2}}$ is not supereulerian. To justify this claim, we denote $V\left(C_{n_{1}}\right)=\left\{v_{11}, v_{12}, \cdots, v_{1 n_{1}}\right\}$, and $V\left(C_{n_{2}}\right)=\left\{v_{21}, v_{22}, \cdots, v_{2 n_{2}}\right\}$. Without loss of generality, we assume that $a_{1}=\left(v_{11}, v_{12}\right)$ and $a_{2}=\left(v_{21}, v_{22}\right)$, and $C_{n_{1}} \oplus_{2} C_{n_{2}}=C_{n_{1}} \oplus_{a_{1}, a_{2}} C_{n_{2}}$. Let $B, B_{1}$ and $B_{2}$ be subdigraphs of $C_{n_{1}} \oplus_{2} C_{n_{2}}$ with $V(B)=\left\{v_{12}\right\}, V\left(B_{1}\right)=\left\{v_{13}\right\}$ and $V\left(B_{2}\right)=\left\{v_{23}\right\}$, respectively. By Lemma 2, we conclude that $C_{n_{1}} \oplus_{2} C_{n_{2}}$ is not supereulerian (see Figure 2).

\section{Sufficient Conditions for Supereulerian 2-Sums of Digraphs}

In this section, we will show several sufficient conditions on $D_{1}$ and $D_{2}$ to assure that the 2-sum $D_{1} \oplus_{2} D_{2}$

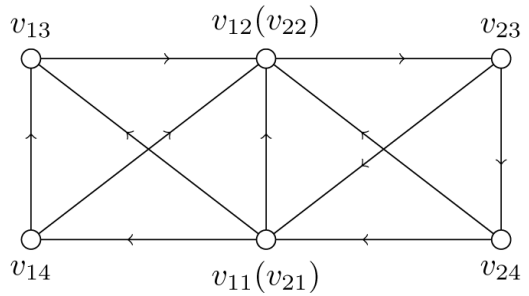

Figure 1. $\lambda\left(D_{1} \oplus_{2} D_{2}\right)=1$ but $\min \left\{\lambda\left(D_{1}\right), \lambda\left(D_{2}\right)\right\}=0$.

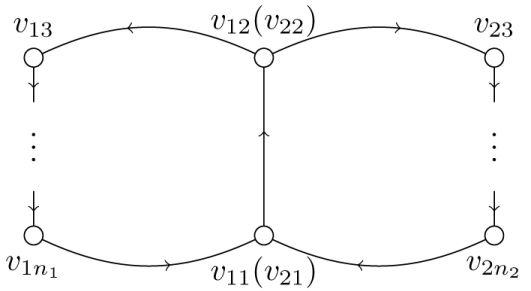

Figure 2. The 2-sum $C_{n_{1}} \oplus_{2} C_{n_{2}}$ of $C_{n_{1}}$ and $C_{n_{2}}$. 
is supereulerian.

Proposition 1 Let $D_{1}$ and $D_{2}$ be two vertex disjoint supereulerian digraphs with $a_{1}=\left(v_{11}, v_{12}\right) \in A\left(D_{1}\right)$ and $a_{2}=\left(v_{21}, v_{22}\right) \in A\left(D_{2}\right)$, and let $D_{1} \oplus_{2} D_{2}$ denote $D_{1} \oplus_{a_{1}, a_{2}} D_{2}$. Each of the following holds.

i) For some $i \in\{1,2\}$, if $D_{i}$ has a spanning eulerian subdigraph $S_{i}$ such that $a_{i} \notin A\left(S_{i}\right)$, then $D_{1} \oplus_{2} D_{2}$ is supereulerian.

ii) If for some $i \in\{1,2\}, D_{i}$ is hamiltonian-connected, then $D_{1} \oplus_{2} D_{2}$ is supereulerian.

Proof. i) Since $D_{1}$ and $D_{2}$ are supereulerian digraphs, $D_{1}$ and $D_{2}$ are strongly connected, and so by Lemma 1, $D_{1} \oplus_{2} D_{2}$ is also strongly connected. Without loss of generality, we assume that $i=1$ and $D_{1}$ has a spanning eulerian subdigraph $S_{1}$ such that $a_{1} \notin A\left(S_{1}\right)$. Since $D_{2}$ is supereulerian, we can pick a spanning eulerian subdigraph $S_{2}^{\prime}$ in $D_{2}$. Then $A\left(S_{1}\right) \cap A\left(S_{2}^{\prime}\right)=\varnothing$ and $V\left(S_{1}\right) \cap V\left(S_{2}^{\prime}\right) \neq \varnothing$. It follows that $D\left[A\left(S_{1}\right) \cup A\left(S_{2}^{\prime}\right)\right]$ is a spanning eulerian subdigraph in $D_{1} \oplus_{2} D_{2}$.

ii) Without loss of generality, we assume that $i=1$ and $D_{1}$ is hamiltonian-connected, and so $D_{1}$ has a $\left(v_{11}, v_{12}\right)$-hamiltonian dipath $T_{1}$ and a $\left(v_{12}, v_{11}\right)$-hamiltonian dipath $T_{2}$. Since $D_{2}$ is supereulerian, $D_{2}$ contains a spanning eulerian subdigraph $S_{2}^{\prime}$. Define

$$
S=\left\{\begin{array}{ll}
D\left[A\left(T_{1}\right) \cup A\left(S_{2}^{\prime}-\left\{\left(v_{21}, v_{22}\right)\right\}\right)\right] & \text { if }\left(v_{21}, v_{22}\right) \in A\left(S_{2}^{\prime}\right) \\
D\left[\left(A\left(T_{2}\right) \cup\left\{\left(v_{11}, v_{12}\right)\right\}\right) \cup A\left(S_{2}^{\prime}\right)\right] & \text { if }\left(v_{21}, v_{22}\right) \notin A\left(S_{2}^{\prime}\right)
\end{array} .\right.
$$

As in any case, $S$ is strongly connected and every vertex $v \in V(S)$ satisfies $d_{S}^{+}(v)=d_{S}^{-}(v)$, and so $S$ is eulerian. Since $V(S)=V\left(T_{i}\right) \cup V\left(S_{2}^{\prime}\right)=V\left(D_{1}\right) \cup V\left(D_{2}\right)$, for $i \in\{1,2\}$, we conclude that $S$ is a spanning eulerian subdigraph of $D_{1} \oplus_{2} D_{2}$, and so $D_{1} \oplus_{2} D_{2}$ is supereulerian.

Theorem 2 [13] If a strict digraph on $n \geq 3$ vertices has $(n-1)^{2}+1$ or more arcs, then it is hamiltonianconnected.

Corollary 1 Let $D_{1}$ be a strict digraph on $n_{1} \geq 3$ vertices and with $\left|A\left(D_{1}\right)\right| \geq\left(n_{1}-1\right)^{2}+1$. If $D_{2}$ is a supereulerian digraph, then $D_{1} \oplus_{2} D_{2}$ is supereulerian.

Proof. By Theorem 2, $D_{1}$ is hamiltonian-connected. Then by Proposition 1 (ii), $D_{1} \oplus_{2} D_{2}$ is supereulerian.

Two classes of supereulerian digraphs seem to be of particular interests in studying supereulerian digraph 2sums. We first present their definitions.

Definition 3.2 Let $D$ be a digraph such that either $D=K_{1}$ or $A(D) \neq \varnothing$. If for any $u, v \in V(D), D$ contains a symmetric dipath from $u$ to $v$, then $D$ is called a symmetrically connected digraph.

Given a digraph $D$, define a relation $\sim$ on $V(D)$ such that $u \sim v$ if and only if $u=v$ or $D$ has a symmetrically connected subdigraph $H$ with $u, v \in V(H)$. By definition, one can routinely verify that $\sim$ is an equivalence relation. Each equivalence class induces a symmetrically connected component of $D$. Hence $D$ is symmetrically connected if and only if $D$ has only one symmetrically connected component. A symmetrically connected component of $D$ is also called a maximal symmetrically connected subdigraph of $D$. When $D$ has more than one symmetrically connected components, we have the following definition.

Definition 3.3 Let $D$ be a weakly connected digraph and $\left\{H_{1}, H_{2}, \cdots, H_{c}\right\}$ be the set of maximal symmetrically connected subdigraphs of $D$ with $c \geq 2$. If for any proper nonempty subset $\mathcal{J} \subset\left\{H_{1}, H_{2}, \cdots, H_{c}\right\}$,

$$
\begin{aligned}
& \text { there exist an } H_{i} \in \mathcal{J} \text {, a vertex } v \in V\left(H_{i}\right) \text {, and an } H_{j} \notin \mathcal{J} \text { such that } \\
& N_{D}^{+}(v) \cap V\left(H_{j}\right) \neq \varnothing \text { and } N_{D}^{-}(v) \cap V\left(H_{j}\right) \neq \varnothing \text {, }
\end{aligned}
$$

then $D$ is partially symmetric.

It is known that both symmetrically connected digraphs and partially symmetric digraphs are supereulerian.

Theorem 3 ([14] and [15]) Each of the following holds.

i) Every symmetrically connected digraph is supereulerian.

ii) Every partially symmetric digraph is supereulerian.

A main result of this section is to show that the digraph 2-sums of symmetrically connected or partially symmetric digraphs are supereulerian.

Lemma 3 Let $D_{1}$ and $D_{2}$ be two vertex disjoint digraphs with $a_{1}=\left(v_{11}, v_{12}\right) \in A\left(D_{1}\right)$ and $a_{2}=\left(v_{21}, v_{22}\right) \in A\left(D_{2}\right)$, and let $D_{1} \oplus_{2} D_{2}$ denote $D_{1} \oplus_{a_{1}, a_{2}} D_{2}$. Each of the following holds.

i) If $D_{1}$ and $D_{2}$ are symmetrically connected, then $D_{1} \oplus_{2} D_{2}$ is symmetrically connected. 
ii) If $D_{1}$ and $D_{2}$ are partially symmetric, then $D_{1} \oplus_{2} D_{2}$ is partially symmetric.

iii) If $D_{1}$ is symmetric and $D_{2}$ is partially symmetric, then $D_{1} \oplus_{2} D_{2}$ is partially symmetric.

Proof. i) For any vertices $x, y \in V\left(D_{1} \oplus_{2} D_{2}\right)$, we shall show that $D_{1} \oplus_{2} D_{2}$ always has a symmetric $(x, y)$ dipath. If for some $i \in\{1,2\}$, we have $x, y \in V\left(D_{i}\right)$, then as $D_{i}$ is symmetrically connected, $D_{i}$ contains a symmetric $(x, y)$-dipath $P$. Since $D_{i}$ is a subdigraph of $D_{1} \oplus_{2} D_{2}, P$ is also a symmetric $(x, y)$-dipath of $D_{1} \oplus_{2} D_{2}$. Hence we may assume that $x \in V\left(D_{1}\right)$ and $y \in V\left(D_{2}\right)$. Since $D_{1}$ and $D_{2}$ are symmetrically connected, $D_{1}$ contains a symmetric $\left(x, v_{11}\right)$-dipath $P_{1}$ and $D_{2}$ contains a symmetric $\left(v_{21}, y\right)$-dipath $P_{2}$. By Definition 2.1, $v_{11}$ and $v_{21}$ represent the same vertex in $D_{1} \oplus_{2} D_{2}$, and so $D_{1} \oplus_{2} D_{2}\left[A\left(P_{1}\right) \cup A\left(P_{2}\right)\right]$ is a symmetric $(x, y)$-dipath in $D_{1} \oplus_{2} D_{2}$.

ii) Fix $i \in\{1,2\}$. Since $D_{i}$ is partially symmetric, for some integer $c_{i}>1$, let $\left\{H_{i 1}^{\prime}, H_{i 2}^{\prime}, \cdots, H_{i c_{i}}^{\prime}\right\}$ be the set of all maximal symmetrically connected subdigraphs of $D_{i}$. Without loss of generality, we assume that $v_{11} \in V\left(H_{11}^{\prime}\right)$ and $v_{21} \in V\left(H_{21}^{\prime}\right)$; and for some $s, t$ with $1 \leq s \leq c_{1}$ and $1 \leq t \leq c_{2}, \quad v_{12} \in V\left(H_{1 s}^{\prime}\right)$ and $v_{22} \in V\left(H_{2 t}^{\prime}\right)$. (We allow the possibility that $s=1$ and/or $t=1$ ). Define, for $1 \leq h \leq c_{1}$ and $1 \leq j \leq c_{2}$,

$$
H_{1 h}=\left\{\begin{array}{ll}
H_{1 h}^{\prime} & \text { if } h \notin\{1, s\} \\
H_{11}^{\prime} \cup H_{21}^{\prime} & \text { if } h=1 \\
H_{1 s}^{\prime} \cup H_{2 t}^{\prime} & \text { if } h=s
\end{array} \text { and } H_{2 j}=\left\{\begin{array}{ll}
H_{2 j}^{\prime} & \text { if } j \notin\{1, t\} \\
H_{11}^{\prime} \cup H_{21}^{\prime} & \text { if } j=1 \\
H_{1 s}^{\prime} \cup H_{2 t}^{\prime} & \text { if } j=t
\end{array} .\right.\right.
$$

Then, $\mathcal{H}=\left\{H_{11}, H_{12}, \cdots, H_{1 c_{1}}, H_{21}, H_{22}, \cdots, H_{2 c_{2}}\right\}$ is the set of all maximal symmetrically connected subdigraphs of $D_{1} \oplus_{2} D_{2}$. Note that $H_{11}=H_{21}$ and $H_{1 s}=H_{2 t}$. We shall show by definition that $D_{1} \oplus_{2} D_{2}$ is partially symmetric. To do that, let $\mathcal{J}$ be a nonempty proper subset of $\mathcal{H}$. We shall show that (3) holds.

Since $\mathcal{H}=\left\{H_{11}, H_{12}, \cdots, H_{1 c_{1}}, H_{21}, H_{22}, \cdots, H_{2 c_{2}}\right\}$, we either have $\mathcal{J} \cap\left\{H_{11}, H_{12}, \cdots, H_{1 c_{1}}\right\} \neq \varnothing$ or $\mathcal{J} \cap\left\{H_{21}, H_{22}, \cdots, H_{2 c_{2}}\right\} \neq \varnothing$. By symmetry, we may assume that $\mathcal{J} \cap\left\{H_{11}, H_{12}, \cdots, H_{1 c_{1}}\right\} \neq \varnothing$.

Suppose first that $\left\{H_{11}, H_{12}, \cdots, H_{1 c_{1}}\right\}-\mathcal{J} \neq \varnothing$. Let $\mathcal{J}^{\prime}=\left\{H_{1 h}^{\prime} \mid H_{1 h} \in \mathcal{J}\right\}$. Then $\left\{H_{11}^{\prime}, H_{12}^{\prime}, \cdots, H_{1 c_{1}}^{\prime}\right\}-\mathcal{J}^{\prime} \neq \varnothing$. Since $D_{1}$ is partially symmetric, there exist an $H_{1 h_{0}}^{\prime} \in \mathcal{J}^{\prime}$, a vertex $v \in V\left(H_{1 h_{0}}^{\prime}\right)$, and an $H_{1 j_{0}}^{\prime} \in\left\{H_{11}^{\prime}, H_{12}^{\prime}, \cdots, H_{1 c_{1}}^{\prime}\right\}-\mathcal{J}^{\prime}$ such that

$$
N_{D_{1}}^{+}(v) \cap V\left(H_{1 j_{0}}^{\prime}\right) \neq \varnothing \text { and } N_{D_{1}}^{-}(v) \cap V\left(H_{1 j_{0}}^{\prime}\right) \neq \varnothing \text {. }
$$

This implies that the vertex $v \in V\left(H_{1 h_{0}}\right), \quad H_{1 h_{0}} \in \mathcal{J}$, and $H_{1 j_{0}} \notin \mathcal{J}$ such that

$$
N_{D_{1} \oplus_{2} D_{2}}^{+}(v) \cap V\left(H_{1 j_{0}}\right) \neq \varnothing \text { and } N_{D_{1} \oplus_{2} D_{2}}^{-}(v) \cap V\left(H_{1 j_{0}}\right) \neq \varnothing .
$$

Thus (3) holds in this case.

Hence we may assume that $\left\{H_{11}, H_{12}, \cdots, H_{1 c_{1}}\right\} \subset \mathcal{J}$. Since $\mathcal{J}$ is a proper subset, we must have $\left\{H_{21}, H_{22}, \cdots, H_{2 c_{2}}\right\}-\mathcal{J} \neq \varnothing$. Since $H_{21}=H_{11} \in \mathcal{J}$, we also have $\left\{H_{21}, H_{22}, \cdots, H_{2 c_{2}}\right\} \cap \mathcal{J} \neq \varnothing$. With a similar argument, we conclude that (3) must also hold in this case.

iii) Let $H_{0}=D_{1}$ and let $\left\{H_{1}^{\prime}, H_{2}^{\prime}, \cdots, H_{c}^{\prime}\right\}$ be the set of all maximal symmetrically connected subdigraphs of $D_{2}$ with $v_{21} \in V\left(H_{1}^{\prime}\right)$ and for some $j \in\{1,2, \cdots, c\}, v_{22} \in V\left(H_{j}^{\prime}\right)$. (We allow the possibility that $j=1$ ). Define

$$
H_{i}=\left\{\begin{array}{ll}
H_{1}^{\prime} \cup H_{0} \cup H_{j}^{\prime} & \text { if } i=1 \text { or } i=j \\
H_{i}^{\prime} & \text { if } i \notin\{1, j\}
\end{array} .\right.
$$

Then $\mathcal{H}=\left\{H_{1}, H_{2}, \cdots, H_{c}\right\}$ is the set of all maximal symmetrically connected subdigraphs of $D_{1} \oplus_{2} D_{2}$. Note that $H_{1}=H_{j}$ with this notation. Let $\mathcal{J}$ be a nonempty proper subset of $\mathcal{H}$. We shall show that (3) holds.

Let $J^{\prime}=\left\{H_{i}^{\prime} \mid H_{i} \in \mathcal{J}\right\}$. Since $\mathcal{J}$ is proper, $\mathcal{J}^{\prime}$ is a nonempty proper subset of $\left\{H_{1}^{\prime}, H_{2}^{\prime}, \cdots, H_{c}^{\prime}\right\}$. Since $D_{2}$ is partially symmetric, by Definition 3.2 , there exist an $H_{i_{0}}^{\prime} \in \mathcal{J}^{\prime}$, a vertex $v \in V\left(H_{i_{0}}^{\prime}\right)$, and an $H_{j_{0}}^{\prime} \in\left\{H_{1}^{\prime}, H_{2}^{\prime}, \cdots, H_{c}^{\prime}\right\}-\mathcal{J}^{\prime}$ such that

$$
N_{D_{1}}^{+}(v) \cap V\left(H_{j_{0}}^{\prime}\right) \neq \varnothing \text { and } N_{D_{1}}^{-}(v) \cap V\left(H_{j_{0}}^{\prime}\right) \neq \varnothing \text {. }
$$


This implies that vertex $v \in V\left(H_{i_{0}}\right), \quad H_{i_{0}} \in \mathcal{J}$ and $H_{j_{0}} \notin \mathcal{J}$ such that

$$
N_{D_{1} \oplus_{2} D_{2}}^{+}(v) \cap V\left(H_{j_{0}}\right) \neq \varnothing \text { and } N_{D_{1} \oplus_{2} D_{2}}^{-}(v) \cap V\left(H_{j_{0}}\right) \neq \varnothing \text {. }
$$

Thus (3) holds, and so by definition, $D_{1} \oplus_{2} D_{2}$ is partially symmetric.

Theorem 4 Let $D_{1}$ and $D_{2}$ be two digraphs. Each of the following holds.

i) If $D_{1}$ and $D_{2}$ are symmetrically connected, then $D_{1} \oplus_{2} D_{2}$ is supereulerian.

ii) If $D_{1}$ and $D_{2}$ are partially symmetric, then $D_{1} \oplus_{2} D_{2}$ is supereulerian.

iii) If $D_{1}$ is symmetric and $D_{2}$ is partially symmetric, then $D_{1} \oplus_{2} D_{2}$ is supereulerian.

Proof. This follows from Theorem 3 and Lemma 3.

It is also natural to consider sufficient conditions on $D_{1}$ and $D_{2}$ for $D_{1} \oplus_{2} D_{2}$ to be hamiltonian.

Theorem 5 If $D_{1}$ is hamiltonian and $D_{2}$ is hamiltonian-connected digraphs, then $D_{1} \oplus_{2} D_{2}$ is hamiltonian.

Proof. Let $V\left(D_{1}\right)=\left\{v_{11}, v_{12}, \cdots, v_{1 n_{1}}\right\}$ with $C=v_{11} v_{12} \cdots v_{1 n_{1}} v_{11}$ be a hamiltonian dicycle of $D_{1}$ and $V\left(D_{2}\right)=\left\{v_{21}, v_{22}, \cdots, v_{2 n_{2}}\right\}$. Let $a_{1}=\left(v_{11}, v_{12}\right) \in A\left(D_{1}\right)$ and $a_{2}=\left(v_{21}, v_{22}\right) \in A\left(D_{2}\right)$, and $D_{1} \oplus_{2} D_{2}=D_{1} \oplus_{a_{1}, a_{2}} D_{2}$. Since $D_{2}$ is hamiltonian-connected, $D_{2}$ contains a $\left(v_{21}, v_{22}\right)$-hamiltonian dipath $P$. Thus $\left(C-\left\{a_{1}\right\}\right) \cup P$ is a hamiltonian dicycle in $D_{1} \oplus_{2} D_{2}$.

Theorem 6 (Thomassen [16]) If a semicomplete digraph $D$ is 4-strong, then $D$ is hamiltonian-connected.

By Theorem 5 and 6, we have the following corollary.

Corollary 2 Let $D_{1}$ and $D_{2}$ be two 4-strong semicomplete digraphs, then $D_{1} \oplus_{2} D_{2}$ is hamiltonian.

\section{Acknowledgements}

The research of Juan Liu was partially supported by grants NSFC (No. 61363020, 11301450) and China Scholarship Council, and the research of Xindong Zhang was supported in part by grants NSFC (No. 11461072) and the Youth Science and Technology Education Project of Xinjiang (No. 2014731003).

\section{References}

[1] Bondy, J.A. and Murty, U.S.R. (2008) Graph Theory. Springer, New York. http://dx.doi.org/10.1007/978-1-84628-970-5

[2] Bang-Jensen, J. and Gutin, G. (2009) Digraphs: Theory, Algorithms and Applications. 2nd Edition. Springer-Verlag, London. http://dx.doi.org/10.1007/978-1-84800-998-1

[3] Boesch, F.T., Suffel, C. and Tindell, R. (1977) The Spanning Subgraphs of Eulerian Graphs. Journal of Graph Theory, 1, 79-84. http://dx.doi.org/10.1002/jgt.3190010115

[4] Pulleyblank, W.R. (1979) A Note on Graphs Spanned by Eulerian Graphs. Journal of Graph Theory, 3, 309-310. http://dx.doi.org/10.1002/jgt.3190030316

[5] Catlin, P.A. (1992) Supereulerian Graphs: A Survey. Journal of Graph Theory, 16, 177-196. http://dx.doi.org/10.1002/jgt.3190160209

[6] Chen, Z.H. and Lai, H.-J. (1995) Reduction Techniques for Super-Eulerian graphs and Related Topics—A Survey. In: Gu, T.-H., Ed., Combinatorics and Graph Theory'95, Vol. 1 (Hefei), World Scientific Publishing, River Edge, 53-69.

[7] Lai, H.-J., Shao, Y. and Yan, H. (2013) An Update on Supereulerian Graphs. WSEAS Transactions on Mathematics, 12, 926-940.

[8] Algefari, M.J. and Lai, H.-J. (2016) Supereulerian Digraphs with Large Arc-Strong Connectivity. Journal of Graph Theory, 81, 393-402. http://dx.doi.org/10.1002/jgt.21885

[9] Bang-Jensen, J. and Maddaloni, A. (2015) Sufficient Conditions for a Digraph to Be Supereulerian. Journal of Graph Theory, 79, 8-20. http://dx.doi.org/10.1002/jgt.21810

[10] Gutin, G. (1993) Cycles and Paths in Directed Graphs. PhD Thesis, School of Mathematics, Tel Aviv University, Tel Aviv-Yafo.

[11] Gutin, G. (2000) Connected (g; f)-Factors and Supereulerian Digraphs. Ars Combinatoria, 54, 311-317.

[12] Hong, Y.M., Lai, H.-J. and Liu, Q.H. (2014) Supereulerian Digraphs. Discrete Mathematics, 330, 87-95. http://dx.doi.org/10.1016/j.disc.2014.04.018

[13] Lewin, M. (1975) On Maximal Circuits in Directed Graphs. Journal of Combinatorial Theory, Series B, 18, $175-179$. http://dx.doi.org/10.1016/0095-8956(75)90045-3 
[14] Algefari, M.J., Alsatami, K.A., Lai, H.-J. and Liu, J. (2016) Supereulerian Digraphs with Given Local Structures. Information Processing Letters, 116, 321-326. http://dx.doi.org/10.1016/j.ipl.2015.12.008

[15] Alsatami, K.A. (2016) A Study on Dicycles and Eulerian Subdigraphs in Digraphs. PhD Dissertation, West Virginia University, Morgantown.

[16] Thomassen, C. (1980) Hamiltonian-Connected Tournaments. Journal of Combinatorial Theory, Series B, 28, $142-163$. http://dx.doi.org/10.1016/0095-8956(80)90061-1 